\title{
APPRIVOISER LA RICHESSE DES COULEURS SUR LA PALETTE DU PEINTRE, OU VERS UNE LISTE TERMINOLOGIQUE DES NOMS DE COULEURS
}

\author{
La couleur n'est pas tant un phénomène naturel \\ qu'une construction culturelle complexe, \\ rebelle à toute généralisation, sinon à toute analyse. \\ Elle met en jeu des problèmes nombreux et difficiles ${ }^{1}$.
}

\section{RICHESSE INATTENDUE}

Paradoxalement, nous commencerons cet article par dire ce qu'il était censé être mais ce qu'il n'est finalement pas et nous essayerons d'en donner la justification. De nombreux travaux cognitifs ou ethnolinguistiques montrent que la conceptualisation des couleurs, telle qu'elle apparaît par le biais des noms de couleurs dans les différentes langues, n'est pas universelle ni homogène ${ }^{2}$, loin de là. Pourtant, dans divers domaines, on essaie de décrire cette catégorie très subjective de la manière la plus objective possible. L'art nous semblait en être un bon exemple : les couleurs se voient attribuer une terminologie bien précise pour que chaque ton se différencie de « son voisin » sur la palette du peintre. Bleu Klein, bleu Matisse, bleu Nattier... ne sont que quelques exemples de ce qui nous

${ }^{1}$ M. Pastoureau, Bleu. Histoire d'une couleur, Éditions du Seuil, Paris 2000, p. 5.

${ }^{2}$ Voir à ce propos notamment l'article d'Anna Wierzbicka : A. Wierzbicka, «Znaczenie nazw kolorów i uniwersalia widzenia », [dans :] eadem, Język - umyst - kultura, wybór prac pod red. Jerzego Bartmińskiego, Państwowe Wydawnictwo Naukowe, Warszawa 1999, pp. 405-450. 
semblait être une volonté de précision et d'univocité. Comme les termes désignant les couleurs dans la peinture, à notre connaissance, n'ont fait jusque-là l'objet d'aucune étude terminologique ni en français ni en polonais, nous avons décidé d'y porter notre regard. Tout au début des recherches sur ce type de termes, nous avons eu un certain pressentiment des problèmes qui pourraient se poser à leur analyse. Parmi les termes désignant les couleurs, une différence se dessinait entre, d'un côté, ceux employés par les historiens de l'art pour décrire les nuances qu'ils perçoivent sur les tableaux et, de l'autre côté, ceux employés par les artistes euxmêmes pour désigner les pigments et les colorants utilisés dans la peinture. Nous nous sommes posée alors comme objectif d'analyser ces deux groupes de termes du point de vue morphologique et, dans une moindre mesure, sémantique, et d'en élaborer une liste terminologique des noms de couleurs afin de pouvoir analyser plus en détails le domaine en question. Mais la réalité a largement dépassé nos attentes et nos ambitions. Et ceci pour deux raisons majeures : en premier lieu, le nombre d'occurrences qu'on a pu recueillir dépasse largement les limites de cet article. En deuxième lieu, les relations sémantiques et conceptuelles entre ces différentes occurrences se sont avérées tellement complexes qu'il serait risqué d'essayer de les décrire toutes. Il a donc fallu faire des choix.

Nous avons décidé, par-dessus tout, de limiter notre corpus des termes étudiés aux termes désignant les pigments. La justification de ce choix principal sera donnée dans la partie suivante de l'article : dans un premier temps, nous envisagerons quelques définitions du phénomène de la couleur pour pouvoir bien saisir sa complexité. Dans un deuxième temps, nous consacrerons quelques mots à la bibliographie du domaine pour pouvoir justifier les choix méthodologiques qu'on a finalement dû faire. Après cette partie « introductive», nous passerons à la partie analytique pour répondre à un double objectif de notre étude : voir quelles sont les caractéristiques formelles des termes de pigments en français et en polonais ainsi qu'examiner les difficultés d'ordre méthodologique qui se posent à l'analyse terminologique de notre corpus.

\section{LE PEINTRE PEINT NON PAS AVEC LES COULEURS DE LA LUMIÈRE MAIS AVEC LES PIGMENTS ${ }^{3}$}

La couleur - en tant que phénomène visuel — a suscité pendant des siècles un intérêt incessant de la part des chercheurs, artistes et philosophes. On a essayé de comprendre sa nature, de la définir. Comme le mentionne Maria Rzepińska, dès l'Antiquité, une question fondamentale a préoccupé les esprits : la couleur, est-elle propriété objective d'un objet ou est-elle une simple impression? Aujourd'hui, on

${ }^{3}$ D'après : J.M. Parramón, Kolor $w$ malarstwie [titre original : The Book of Color], trad. D. Ostrowska-Dziuba, S. Ostrowski, Wydawnictwa Szkolne i Pedagogiczne, Warszawa 1995, p. 53. 
sait que la couleur est un phénomène fort complexe, physique, physico-chimique, physiologique et psychologique à la fois ${ }^{4}$. Yves Charnay et Hélène de Givry donnent une définition ( « la plus juste») de la couleur qui réunit tous ces points de vue : c'est « une impression ressentie par l'œil au contact d'ondes électromagnétiques constitutives de la lumière $»^{5}$. Et plus loin, ils expliquent : « La couleur est une sensation. Des récepteurs situés dans l'œil captent les radiations lumineuses, les transforment en signaux électrochimiques et les transmettent au cerveau. Là, selon leur longueur d'onde, ces stimuli deviennent des sensations colorées $»^{6}$. À part cette dimension physique et physiologique des couleurs, il ne faut pas oublier leur dimension culturelle et sociale ${ }^{7}$... Par conséquent, les couleurs — avec toutes leurs facettes - sont l'objet de recherches de divers spécialistes. C'est donc avec raison que les auteurs du guide Comment regarder... les couleurs dans la peinture distinguent trois types de couleurs : 1) « couleurs spectrales, issues de la lumière »; 2) «couleurs pigmentaires, provenant de matériaux, dites aussi "couleurs matières" "; et 3) " couleurs imaginaires, résultant d'une interprétation de notre cerveau $\rangle^{8}$. En nous basant sur cette typologie, pour la suite de notre étude, nous faisons une nette distinction terminologique entre les couleurs, c'est-à-dire des " sensations colorées » qu'on perçoit (aussi bien du point de vue physique que psychologique), et les pigments (les « couleurs pigmentaires »), c'est-à-dire les matériaux qui communiquent leurs couleurs aux supports sur lesquels ils sont utilisés?.

Avant de passer à l'analyse des termes désignant les pigments, il convient de s'attarder sur la bibliographie extrêmement riche consacrée au sujet des couleurs dans le sens large du mot. Les définitions présentées confirment notre pressentiment que, lorsqu'on parle des couleurs, on peut avoir, au moins, deux notions en

${ }^{4}$ M. Rzepińska, Historia koloru w dziejach malarstwa europejskiego, Wydawnictwo Arkady, Warszawa 2009, p. 8.

${ }^{5}$ Y. Charnay, H. de Givry, Comment regarder... les couleurs dans la peinture, série « Clés et repères », Éditions Hazan, Paris 2011, p. 4.

${ }^{6}$ Ibidem, p. 10.

${ }^{7}$ Rzepińska rappelle le dualisme cartésien et la différence qui en résultait entre les sciences naturelles et exactes, d'une part, et les sciences humaines, d'autre part. La couleur en tant qu'objet de recherches était (et reste) très exceptionnelle puisqu'elle se trouvait dans ces deux mondes. Son statut pourrait être représenté d'une manière symbolique par l'opposition entre deux théories de la couleur : celle de Goethe qui a adopté une vision physiologique et psychologique des couleurs, et celle de Newton, purement physique, qui a mené à la découverte du fameux spectre lumineux (M. Rzepińska, op. cit., p. 9).

8 Y. Charnay, H. de Givry, op. cit., p. 10.

${ }^{9}$ Bernard Guineau remarque que le terme pigment est un terme normalisé qui, depuis le début du XX $X^{\mathrm{e}}$ siècle, a remplacé celui plus ancien de couleur. Il désigne « un matériau colorant, de nature minérale ou organique, solide (...), insoluble dans les milieux de suspension usuels et capable de communiquer sa couleur au milieu qui l'entoure » (B. Guineau, Glossaire des matériaux de la couleur et des termes techniques employés dans les recettes de couleurs anciennes, Brepols Publishers, Turnhout 2005, p. 558). 
tête : les noms de couleurs en tant qu'objets de perception, et les noms de couleurs en tant que matériaux utilisés en peinture, en teinture, etc. Si on parcourt la liste des ouvrages consacrés aux couleurs, cette dichotomie semble se confirmer ${ }^{10}$. D'un côté, on a donc tout un éventail d'ouvrages qui traitent de la perception des couleurs et de leur dénomination dans les différentes langues : pour le français, on peut évoquer les dictionnaires consacrés aux différentes couleurs d'Annie Mollard-Desfour, notamment le plus récent, de 2008, consacré au blanc ${ }^{11}$. Pour le polonais, il s'agit entre autres de la monographie de Ryszard Tokarski qui, en adoptant un point de vue interdisciplinaire, analyse le sens des lexèmes de couleurs dans la langue polonaise d'aujourd'hui ${ }^{12}$. Il ne faut pas oublier non plus les travaux cognitifs sur les couleurs et leur perception rendue dans différentes langues. Pour ne mentionner que les travaux les plus « classiques », citons ceux $\mathrm{d}^{\prime}$ Anna Wierzbicka ${ }^{13}$ ou de Brent Berlin et Paul Kay ${ }^{14}$. On pourrait y ajouter encore le recueil d'articles dirigé par Serge Tornay, à visée ethnolinguistique ${ }^{15}$.

De l'autre côté, nous avons des ouvrages dont les auteurs s'occupent des couleurs et des pigments du point de vue de l'histoire de l'art et des techniques picturales. Pour le polonais, l'ouvrage de référence, réédité déjà plusieurs fois, est la monographie de Maria Rzepińska sur l'histoire de la couleur dans la peinture européenne ${ }^{16}$. Pour le français, on pourrait citer le remarquable glossaire ${ }^{17}$ de Bernard Guineau ${ }^{18}$ sur lequel nous reviendrons dans la suite, la monographie sur la théorie de la couleur dans la peinture de Georges Roque ${ }^{19}$ ou une bonne introduction au

${ }^{10}$ Toutes les catégories thématiques décrites dans ce paragraphe sont représentées aussi bien dans les ouvrages de vulgarisation que dans les textes scientifiques.

11 A. Mollard-Desfour, Le Blanc. Dictionnaire de la couleur. Mots et expressions d'aujourd'hui. $X X^{e}-X X I^{e}$ siècles, CNRS Éditions, Paris 2008.

${ }^{12}$ R. Tokarski, Semantyka barw we wspótczesnej polszczyźnie, Wydawnictwo Uniwersytetu Marii Curie-Skłodowskiej, Lublin 2004.

${ }^{13}$ Par ex. : A. Wierzbicka, op. cit.

${ }^{14}$ Notamment : B. Berlin, P. Kay, Basic Color Terms. Their Universality and Evolution, University of California Press, Berkeley \& Los Angeles 1969.

15 S. Tornay (dir.), Voir et nommer les couleurs, CNRS, Nanterre 1978.

${ }^{16}$ M. Rzepińska, op. cit.

17 B. Guineau, op. cit.

${ }^{18}$ Il faut souligner — sans porter de jugement — que l'auteur de ce glossaire n'est pas linguiste mais spécialiste du domaine. Sur le site de l'éditeur, on peut lire : «Bernard Guineau n'est pas seulement un pionnier dans l'application des méthodes physico-chimiques aux pigments et aux colorants anciens. Il a aussi scruté la littérature technique de la couleur depuis l'Antiquité jusqu'au $\mathrm{XX}^{\mathrm{e}}$ siècle. Le présent dictionnaire résulte d'immenses recherches documentaires. Il fournit l'identification, et très souvent la formule chimique de plusieurs milliers de termes anciens relatifs à la couleur et aux matériaux de la couleur. C'est un outil indispensable pour l'historien d'art, l'historien des sciences, l'analyste, mais aussi le restaurateur et l'artisan » (<http://www.brepols.net/Pages/ ShowProduct.aspx?prod_id=IS-9782503516431-1> [consulté le 28.09.2015]).

${ }^{19}$ G. Roque, Art et science de la couleur. Chevreul et les peintres, de Delacroix à l'abstraction, Éditions Gallimard, Paris 2009. 
sujet des couleurs dans la peinture d'Yves Charnay et Hélène de Givry ${ }^{20}$. Pour clore cette sélection (par nécessité très réduite) des ouvrages consacrés à cette thématique, il est nécessaire de mentionner encore les livres dans lesquels la couleur est envisagée surtout en tant que phénomène culturel et social : nous n'évoquerons ici que les livres passionnants de Michel Pastoureau ${ }^{21}$ ou les très nombreux ouvrages de vulgarisation qui se concentrent sur la symbolique des couleurs d'une culture à l'autre, comme L'intelligence de la couleur d'Élisabeth Brémond ${ }^{22}$. Soulignons que le point de vue linguistique, même s'il apparaît nécessairement dans les deux derniers types d'ouvrages présentés, n'est pas une fin en soi dans ces ouvrages.

$\mathrm{Vu}$ la distinction présentée dans le premier paragraphe, il nous semble inutile de mélanger toutes les approches présentées et de vouloir décrire tous les usages des noms de couleurs à la fois. Pour suivre la visée terminologique de notre travail, nous avons choisi de ne garder pour la constitution de notre corpus que les ouvrages de la deuxième catégorie présentée supra, c'est-à-dire les ouvrages écrits par des spécialistes de l'art pour des spécialistes de l'art ${ }^{23}$. En nous basant sur ces ouvrages, nous avons tenté de recueillir les termes désignant, comme cela a déjà été souligné, les pigments utilisés en peinture. Une tentative qui reste cependant inachevée, puisque le nombre de ce type de termes dépasse largement le cadre de cette étude ${ }^{24}$, mais qui, en même temps, ouvre des perspectives pour d'autres futures études. À ce stade des recherches, notre corpus des termes français et polonais est divisé selon les six couleurs répertoriées par Léonard de Vinci dans son Traité de la peinture : blanc, jaune, vert, bleu, rouge et noir ${ }^{25}$. La liste des sources de termes est pour le moment très restreinte, mais en même temps, elle nous a semblé suffisante pour construire un inventaire provisoire des termes désignant les pigments. En constituant le corpus, nous avons travaillé séparément pour les deux langues. Toutefois, l'ouvrage qui nous a servi de base théorique et de point de départ pour nous initier au domaine a été le glossaire français de Guineau ${ }^{26}$. Pour cet article, finalement, nous avons renoncé à y ajouter la liste des termes de pigments parce que leur nombre est si considérable qu'il nous forcerait à opérer des choix à notre avis discutables. Pour le moment, il nous semble plus important de présenter les mécanismes morphologiques et motivationnels qui régissent la création de ce type de termes ainsi que de signaler les problèmes généraux qu'on peut rencontrer en analysant la terminologie des pigments.

${ }^{20}$ Y. Charnay, H. de Givry, op. cit.

${ }^{21}$ Voir notamment M. Pastoureau, op. cit.

22 E. Brémond, L'intelligence de la couleur, Albin Michel, Paris 2002.

${ }^{23}$ C'est à dessein que nous utilisons ici une expression assez large pouvant englober les différentes professions liées à l'art (les artistes eux-mêmes, les historiens de l'art, les techniciens de l'art, etc.).

${ }^{24}$ Pour donner une idée de l'ampleur de cette terminologie, il suffit de dire que Guineau récense plus de 100 termes pour les seuls pigments blancs, sans parler des autres couleurs (B. Guineau, op. cit., pp. 116-129).

25 Y. Charnay, H. de Givry, op. cit., p. 10.

26 B. Guineau, op. cit. 


\section{LA PALETTE DU PEINTRE À TRAVERS LE PRISME DE LA LANGUE}

Pour pouvoir décrire le «tableau » des termes de pigments, riche en « couleurs et leurs nuances ", observons d'abord la palette du peintre du point de vue purement linguistique. L'analyse dérivationnelle et, surtout, motivationnelle qui suit n'est que provisoire mais permet de montrer - sans trop entrer dans les détails les mécanismes les plus fréquents qui régissent la création des termes de pigments. Les constatations de Denis Apothéloz qu'" aucun mot n'est jamais créé ex nihilo, à partir d'une simple concaténation de phonèmes» et que « les procédés de formation du vocabulaire (...) aboutissent presque toujours à donner au mot nouveau une origine reconnaissable ${ }^{27}$ se confirment nettement. Lorsqu'on regarde la forme de ces termes, on s'aperçoit que sa motivation relève de quatre catégories majeures :

\section{A) ORIGINE NATURELLE OU SYNTHÉTIQUE DU PIGMENT}

Ce type de motivation est employé très souvent. Il semble juste et naturel de vouloir utiliser le nom de la substance (chimique ou autre) pour former le nom de pigment qui en est extrait. Ainsi, en français, on a un groupe extrêmement nombreux de termes construits de la manière suivante : $\mathrm{N}_{\text {couleur }}+$ Prép $+\mathrm{N}_{\text {substance }}$ - comme blanc de plomb, bleu de chrome, brun de cuivre, jaune de cadmium, noir d'antimoine et vert de cobalt, pour ne mentionner que quelques exemples. En polonais, la tendance est la même, sauf que le complément prépositionnel est remplacé par l'adjectif équivalent : $\mathrm{N}_{\text {couleur }}+\mathrm{Adj}_{\text {substance }}$ - biel ołowiana ('blanc de plomb'), żółcień kobaltowa ('jaune de cobalt'), czerwień żelazowa ('rouge de fer'), czerń manganowa ('noir de manganèse'), etc.

Remarquons seulement que le deuxième élément du terme n'est pas nécessairement formé à partir d'une substance d'origine chimique. Il peut aussi bien désigner l'origine animale ou végétale du pigment, comme dans les termes blanc de baleine (substance obtenue à partir de la graisse de baleine), bleu de sureau (laque bleue extraite des baies de sureau noir), brun de chicorée ou le fameux indigo (au départ extrait des feuilles et des tiges des indigotiers).

\section{B) ORIGINE GÉOGRAPHIQUE DU PIGMENT}

L'origine géographique du pigment inspire la formation de termes aussi fréquemment que la première catégorie. Les entités utilisées sont les noms de villes, de régions ou de pays dans lesquels les pigments ont été fabriqués. La structure des termes français est double:

27 D. Apothéloz, La construction du lexique français. Principes de morphologie dérivationnelle, Éditions Ophrys, Paris 2002, p. 16. 
- $\mathrm{N}_{\text {couleur }}+$ Prép $+\mathrm{N}_{\text {lieu }}$ comme dans les termes brun de Breslau (pigment fabriqué dans les usines chimiques de Breslau au XIX ${ }^{\mathrm{e}}$ siècle), céruse de Rotterdam, rouge d'Andrinople, rouge de Chine, vert de Prusse ;

- $\mathrm{N}_{\text {couleur }}+\mathrm{Adj}_{\text {lieu }}$ comme dans les termes blanc anglais, bleu égyptien, bleu persan, noir indien ou vert turc.

Pour le polonais, cette fois encore, la structure de ce type de termes est la suivante : $\mathrm{N}_{\text {couleur }}+\mathrm{Adj}_{\text {lieu }}$ comme dans les termes biel kremska ('blanc de Krems'), biel wenecka ('blanc de Venise'), żótcień neapolitańska ('jaune de Naples'), czerwień hiszpańska ('rouge d'Espagne') ou błękit paryski ('bleu de Paris').

Certains termes moins transparents font partie de cette catégorie, tel, en polonais, kaolin ou son synonyme glinka kaolinowa, et en français, kaolin ou son synonyme blanc de Chine, qui désignent un pigment d'origine naturelle obtenu à partir des argiles extraites des carrières chinoises des Gao-ling, ce qui signifie les 'Collines Hautes'.

\section{C) INVENTEUR OU UTILISATEUR DU PIGMENT}

Dans cette troisième catégorie, nous avons recensé les termes complexes dont le deuxième élément est le nom de la personne qui a inventé ou tout simplement utilisé le pigment donné. Dans les deux langues, la structure de ces termes est similaire : en français, le nom de la couleur est suivi d'un complément prépositionnel $\mathrm{N}_{\text {couleur }}+$ Prép $+\mathrm{N}_{\text {personne }}$ ou uniquement du nom propre $\mathrm{N}_{\text {couleur }}+\mathrm{N}_{\text {personne }}$; en polonais, le complément du nom est décliné au génitif $\mathrm{N}_{\text {couleur }}+\mathrm{N}_{\text {personne (gén.). }}$.

En ce qui concerne les personnes dont les noms sont utilisés dans les termes, ce sont :

- soit les industriels ou les savants qui ont inventé ou fabriqué la substance chimique ou le pigment, comme dans les termes blanc de Griffith, bleu Guimet, bleu Monthiers ou błękit Peligota ('bleu de Péligot');

- soit les peintres dont les œuvres sont marquées par l'utilisation d'un pigment donné : brun Rubens, jaune Véronèse, brąz van Dycka ('brun van Dyck'), róż Van Dycka ('rose van Dyck'), zieleń Guigneta ('vert Guignet'), zieleń Veronese'a ('vert Véronèse'), etc.

\section{D) ASSOCIATIONS DIVERSES}

La dernière catégorie regroupe les termes dont le deuxième élément vient d'associations diverses à des entités dont la couleur est caractéristique. La couleur du pigment peut ainsi être associée à celle d'une planète (brun de Mars, jaune de Mars, czerwień Marsa ('rouge de Mars'), róż Saturna ('rose de Saturne')), d'un fruit (zótcień cytrynowa ('jaune citron')), d'une boisson (blanc de lait), du ciel (bleu azur, bleu céleste, btękit nieba ('bleu de ciel')), de l'eau (zieleń lazurowa 
('vert azur')), d'une pierre précieuse (blanc perle, bleu de lapis-lazuli, zieleń szmaragdowa ('vert émeraude')), etc.

Pour terminer cette partie, remarquons que cette diversité des catégories motivationnelles explique le nombre considérable de synonymes dans cette terminologie. On pourrait cependant se poser la question du statut théorique de ces synonymes : les termes désignant le même type de pigment mais indiquant des origines géographiques différentes sont-ils vraiment synonymes?

\section{DERRIÈRE LA PALETTE DU PEINTRE}

Bernard Guineau, dans 1'introduction de son ouvrage ${ }^{28}$, signale certains problèmes auxquels il a dû faire face en travaillant sur la constitution de son glossaire. Il est essentiel de les présenter ici si on veut bien comprendre le caractère complexe, chargé de connotations, de la terminologie des pigments. À travers tous ces termes, la palette du peintre peut apparaître comme un véritable symbole de l'histoire et de la culture. Sans en avoir peut-être le pressentiment, nous entrons dans une réalité fortement marquée par la culture, par l'époque avec ses événements, par l'histoire de la science, par la géographie, etc. Nous allons donc présenter quelques unes des difficultés évoquées par Guineau en les illustrant avec une couleur choisie - le blanc - et les termes français désignant les pigments qui permettent de l'obtenir ${ }^{29}$. Notre objectif est de voir comment le fonctionnement très complexe des termes désignant les pigments peut entraîner, à l'analyse, beaucoup de difficultés méthodologiques aussi bien pour les spécialistes de l'art que pour les terminologues.

\section{A) TERMES PROPRES À UNE ÉPOQUE OU À UNE AIRE CULTURELLE PARTICULIÈRE}

Les exemples de ce type abondent. Le blanc de chlorure de plomb, entre autres, est un pigment blanc, composé de chlorure basique de plomb, qui a été employé en peinture d'abord en Extrême-Orient, à partir du XIII et jusqu'au XV siècle, puis en Occident, au cours du XIX ${ }^{\mathrm{e}}$ siècle.

Guineau fait remarquer également que le passage des termes d'une époque à l'autre a très souvent été lié à une transmission de savoirs fautive : "Cette variété de termes traduit aujourd'hui de très anciennes appropriations faites de translittérations ou de traductions parfois incertaines, sinon hasardeuses $»^{30}$.

28 B. Guineau, op. cit., pp. 9-10.

29 Ibidem, pp. 116-129.

30 Ibidem, p. 9. 


\section{B) TERMES ISSUS D’UNE AIRE GÉOGRAPHIQUE PARTICULIÈRE}

Il arrivait très fréquemment qu'un même type de pigment soit fabriqué dans des zones géographiques différentes. Ainsi, le blanc de craie en tant que pigment blanc était obtenu à partir de calcaires de diverses origines, par exemple du Bassin Parisien. On distinguait alors la craie extraite des carrières des environs de Paris : ce type de pigment, selon son origine exacte, était désigné par les termes blanc de Paris, blanc de Meudon ou blanc de Bougival. Un autre type de blanc de craie (réputée la plus blanche) venait de la région de Champagne et était désigné par deux termes : blanc de Troyes ou blanc de Champagne. Le troisième type de blanc de craie était extrait en Normandie et désigné par les termes blanc de Rouen et blanc de Dieppedalle. Pour compliquer encore ce tableau, on peut ajouter à la liste des blancs de craie celui obtenu en Espagne, utilisé à partir du XVII ${ }^{\mathrm{e}}$ ou du XVIII ${ }^{\mathrm{e}}$ siècle, et appelé blanc d'Espagne.

Un autre exemple très intéressant parmi les pigments blancs est le cas du blanc de plomb qui était fabriqué en différents endroits, et en plus, par différentes techniques. Ainsi, un type de blanc de plomb était fabriqué en Allemagne, dans la ville de Krems, selon le procédé dit de «Krems », et il était désigné par le terme - nomen est omen - blanc de Krems. Ce terme risque d'être confondu avec un autre - le blanc de Kremnitz - qui désigne également un blanc de plomb mais fabriqué selon un autre procédé et à un autre endroit : à Kremnitz, près de Vienne (et sans surprise donc, le terme blanc de Vienne est son synonyme).

\section{C) DIVERSITÉ DES EMPLOIS DU TERME}

La peinture est une forme d'art dans laquelle les artistes peuvent se servir de différentes techniques picturales. Il va de soi que chaque technique va utiliser ses propres matériaux, dont les pigments. De même, certains pigments trouveront un emploi non seulement dans la peinture, mais également dans la teinture ou dans d'autres formes d'art. Ainsi, le terme blanc d'étain peut désigner plusieurs types de pigments utilisés notamment dans la peinture à l'huile, pour la faïence, dans l'enluminure, dans l'aquarelle (mais uniquement au XVII ${ }^{\mathrm{e}}$ siècle) ou dans les glaçures céramiques.

En outre, certains matériaux de la couleur ne sont pas employés uniquement dans le domaine de l'art, mais aussi « comme attributs symboliques les plus variés ». Nombreuses étaient ( « étaient» puisque cela est vrai surtout pour les produits anciens) les substances reconnues pour leurs propriétés thérapeutiques et employées en tant que médicaments, inscrites dans la pharmacopée ancienne, dont les extraits trouvaient une utilisation dans la peinture ou dans la teinture en tant que colorants ou pigments. Dans ces cas, les substances étaient désignées par de nombreux termes, les uns renvoyant uniquement au côté thérapeutique des substances, les autres étant utilisés pour nommer les pigments. Dans le glossaire 
de Guineau, on peut trouver de nombreux exemples de ce type de diversité terminologique. Prenons comme exemple l'antimoine qui, comme métal, trouvait de nombreux emplois dans la pharmacopée ancienne : il était désigné par des termes comme fleurs argentines d'antimoine, neige argentine d'antimoine ou antimoine diaphorétique. Dans les arts, il ne sera employé qu'au $\mathrm{XX}^{\mathrm{e}}$ siècle sous la forme d'un pigment blanc artificiel appelé blanc d'antimoine.

En ce qui concerne la diversité des emplois d'un même terme, nombreux sont les cas de confusion entre le matériau et sa couleur : le terme désigne donc « à la fois un matériau et la couleur qui caractérise ce matériau ». Le terme blanc d'argent désigne, ainsi, soit la couleur blanche vive (et brillante comme l'argent), soit deux sortes (!) de pigments blancs de plomb qui se caractérisent par une composition chimique différente : le premier s'appelle blanc de plomb et est fabriqué en Allemagne (synonyme : blanc de Krems), et l'autre ne comporte pas de charge minérale blanche (ses synonymes seraient blanc de baryte ou blanc de craie).

\section{D) CHANGEMENTS DE SENS}

Revenons encore à un pigment qui a été déjà évoqué, le blanc d'Espagne. Comme le fait remarquer Guineau, c'est un terme à « risques de confusions possibles» : avant le XVII ${ }^{\mathrm{e}}$ siècle, il désignait un type de blanc de plomb; après le XVII ${ }^{\mathrm{e}}$ par contre, il a été utilisé pour désigner un type de blanc de craie extrait dans la péninsule Ibérique.

Si on rassemble tous les facteurs qui viennent d'être présentés, on est confronté à une terminologie dans laquelle, du point de vue d'un lexicologue ou d'un terminologue, les problèmes sont multiples : « (...) des mots différents, parfois nombreux, désignent la même couleur ou le même colorant. Inversement, il arrive qu'un seul mot se rapporte à divers pigments ou colorants. Il faut aussi savoir si un terme désigne une couleur ou le produit qui a permis de l'obtenir $»^{31}$. Au final, on a donc un vaste ensemble de termes qui voyagent à travers les époques et les styles, qui s'approprient de nouveaux candidats et en abandonnent d'autres. C'est une des terminologies les moins ordonnées possible où les synonymes, homonymes et termes polysémiques sont pléthore ${ }^{32}$.

\section{CONCLUSIONS}

Derrière la palette du peintre, nous avons découvert l'existence d'une terminologie extrêmement riche et complexe qui est une belle manifestation de l'histoire des cultures et des arts. L'analyse de la terminologie des pigments a démontré

${ }^{31}$ Ibidem, p. 7.

32 Pour la terminologie traditionnelle, issue de l'école de Vienne, ce serait un véritable cauchemar! 
également à quel point l'art est lié à la technique et à la science. L'ouvrage de Georges Roque déjà évoqué porte d'ailleurs le titre Art et science de la couleur $^{33}$. La liste de pigments et leur composition chimique peuvent faire penser au tableau de Mendeleïev. Pour peindre un tableau, il faut bien choisir les pigments et, surtout, il faut savoir les utiliser. Comme l'écrivait Léonard de Vinci dans ses Carnets : "Ceux qui se vouent à la pratique, sans la science de la couleur, sont comme le pilote qui s'embarquerait sans timon, ni boussole et ne savent jamais dire, à coup sûr, où ils vont ${ }^{34}$. Henri Matisse ajoutait : «Faire de l'ordre dans ses couleurs, c'est mettre de l'ordre dans ses idées. Rien n'empêche de composer avec quelques couleurs, comme la musique, qui est bâtie uniquement sur sept notes (...). De même que la modification de l'expression musicale peut venir d'un tout petit rien, il y a autant de différences entre les tons majeurs et les tons mineurs qu'entre le soleil et son ombre $»^{35}$.

La terminologie des pigments semble ouvrir de nouvelles perspectives pour des études terminologiques, et ceci aussi bien du point de vue cognitif que diachronique. Normalement, dans des langues de spécialité, certains termes apparaissent, d'autres disparaissent lorsqu'ils ne sont plus utilisés ou si les objets auxquels ils renvoient n'existent plus. La situation des termes de pigments semble assez particulière dans cette matière. Effectivement, certains pigments ne sont plus utilisés à certaines époques (pour des raisons de mode ou à cause de leur toxicité ou d'autres propriétés) mais cela ne signifie pas nécessairement que les termes qui les désignent sont voués à la disparition ou à l'oubli, loin de là. Leur trace reste dans les œuvres artistiques, ce qui garantit en quelque sorte leur survie.

Pour terminer, revenons à la citation de Michel Pastoureau qui ouvre notre contribution. Dans son ouvrage, l'auteur souligne que « la couleur est d'abord un fait de société », et il ajoute tout de suite : « il n'y a pas de vérité transculturelle de la couleur $»^{36}$. Par conséquent, la seule démarche qui s'impose pour l'étudier, c'est la démarche historique, qui est la seule susceptible de démontrer en toute complexité que : "C'est la société qui "fait" la couleur, qui lui donne sa définition et son sens, qui construit ses codes et ses valeurs, qui organise ses pratiques et détermine ses enjeux $»^{37}$. Il nous semble que la même exigence s'impose quant à l'étude terminologique des noms de pigments. L'approche diachronique — de plus en plus représentée en terminologie en tant que discipline — n'est pas seulement souhaitable pour apporter des informations intéressantes, mais même obligatoire pour pouvoir bien faire la distinction entre les synonymes, pour décrire adéquatement l'usage de certains termes, bref, pour ne rien perdre de cette richesse cachée derrière les termes.

\footnotetext{
${ }^{33}$ G. Roque, op. cit.

34 E. Brémond, op. cit., p. 239.

35 Ibidem.

${ }^{36}$ M. Pastoureau, op. cit., p. 5.

${ }^{37}$ Ibidem, p. 8 .
} 


\title{
SOURCES DE TERMES
}

\section{POLONAISES}

M. Rzepińska, Historia koloru w dziejach malarstwa europejskiego, Wydawnictwo Arkady, Warszawa 2009.

J. Werner, Podstawy technologii malarstwa i grafiki, Państwowe Wydawnictwo Naukowe, Warszawa 1989.

\section{FRANÇAISES}

B. Guineau, Glossaire des matériaux de la couleur et des termes techniques employés dans les recettes de couleurs anciennes, Brepols Publishers, Turnhout 2005.

Y. Charnay, H. de Givry, Comment regarder ... les couleurs dans la peinture, série " Clés et repères », Éditions Hazan, Paris 2011.

\section{HOW TO MASTER THE ABUNDANCE OF COLOURS ON THE PAINTER'S PALETTE, OR A STEP TOWARDS THE TERMINOLOGICAL LIST OF TERMS RELATED TO COLOURS}

\author{
Summary
}

Colour as a subject has led to numerous studies in various disciplines (such as physics, psychology, chemistry or history of art, to name a few). In the field of linguistics, the names of colours have been analysed from different points of view but all those works concerned mostly the words related to colours used in the general language. The aim of the present paper is to focus on the terms related to colours used in history of art texts, and to show the unexpected complexity of such terminology. After presenting the definitions of colour we will describe two different aspects of French terminology related to colour: firstly, the forms of terms from the point of view of their morphological and semantic motivation, and secondly, the cultural and historical reasons of such linguistic abundance and complexity of the colours' terminology.

Key words: terminology, painting, pigments, colour. 